\section{Doença Renal do Diabetes: A Importância do Diagnóstico e Tratamento Precoces}

\author{
Diabetes kidney disease: the importance of early \\ diagnosis and treatment
}

Ana Carolina Rizzato, Vanessa Resende Souza Silva e Aline de Brito Soyer.

\section{RESUMO:}

A nefropatia diabética (ND) é a principal causa de terapia de substituição renal nos países desenvolvidos e no Brasil é a segunda causa de necessidade de diálise, ficando atrás da hipertensão arterial. A identificação precoce de alterações como a diminuição da taxa de filtração glomerular e a albuminúria são cruciais para prevenir a evolução da doença. No tratamento, além do controle glicêmico, alguns fármacos para controle da pressão arterial são empregados para tratar ou mesmo retardar o desenvolvimento da ND, tais como os inibidores da ECA e os bloqueadores da angiotensina II. Há ainda os fármacos mais recentes, inibidores da SGLT2, utilizados no tratamento do diabetes que estão sendo estudados com evidências de nefroproteção e impedirem a progressão da doença. 0 objetivo deste trabalho foi conhecer uma das principais causas de morbidade e mortalidade em indivíduos com Diabetes Mellitus, a importância de seu diagnóstico precoce e identificar os possíveis tratamentos preventivos mais recentes como forma de não evoluir para doença renal, com enfoque nos inibidores da SGLT2. A metodologia utilizada foi a revisão literária com busca nas bases de dados Scielo, PUBMED, LILACS, MEDLINE E BVS. Os resultados comprovaram a segurança e eficiência dos inibidores da SGLT2 como adjuvantes no tratamento da ND. Os benefícios superam os riscos e medicamentos como a dapagliflozina, empagliflozina e canagliflozina devem ser prescritos aos pacientes diabéticos.

PALAVRAS-CHAVE: Nefropatias Diabéticas; Complicações do Diabetes; Inibidores do Transportador 2 de Sódio-Glicose.

\section{ABSTRACT}

Diabetic nephropathy (DN) is the main cause of renal replacement therapy in developed countries and in Brazil it is the second cause of need for dialysis, behind arterial hypertension. Early identification of changes such as decreased glomerular filtration rate and albuminuria are crucial to prevent disease progression. In the treatment, in addition to glycemic control, some drugs to control blood pressure are used to treat or even delay the development of DN, such as ACE inhibitors and angiotensin II blockers. There are also the most recent drugs, SGLT2 inhibitors, used in the treatment of diabetes that are being studied with evidence of nephroprotection and to prevent the progression of the disease. The objective of this study was to find out one of the main causes of morbidity and mortality in individuals with Diabetes Mellitus, the importance of their early diagnosis and to identify the most recent possible preventive treatments as a way of not progressing to kidney disease, with a focus on SGLT2 inhibitors. The methodology used was the literary review with search in the databases Scielo, PUBMED, LILACS, MEDLINE AND VHL. The results confirmed the safety and efficiency of SGLT2 inhibitors as adjuvants in the treatment of DN. The benefits outweigh the risks and drugs such as dapagliflozin, empagliflozin and canagliflozin should be prescribed to diabetic patients.

KEYWORDS: Diabetic Nephropathies; Diabetes Complications; Sodium-Glucose Transporter 2 Inhibitors. 


\section{INTRODUÇÃO}

A prevalência do Diabetes Mellitus tipo 2 (DM2) tem aumentado principalmente em países em desenvolvimento, sendo a principal causa de necessidade de terapia de substituição renal (TRS) nesses países e a segunda causa no Brasil ${ }^{1}$. Os fatores responsáveis por esse aumento da doença são o envelhecimento da população, o processo de urbanização, a crescente prevalência da obesidade e sedentarismo, bem como a maior sobrevida dos pacientes com DM devido ao diagnóstico precoce e tratamento efetivo².

Por se tratar de uma doença crônica, as implicações da doença afetam diretamente a qualidade de vida do paciente e de sua família, além de ser onerosa tanto para o portador quanto para o Estado. Os indivíduos que necessitam de terapia de substituição renal precisam se adaptar à nova rotina, assim como as pessoas que convivem com ele, o que pode ser desgastante para o doente e para a família ${ }^{3}$.

A doença renal do diabetes é uma complicação crônica microvascular frequente que acomete entre 30 a $50 \%$ dos pacientes com DM e elevada excreção urinária de albumina². Em alguns casos o paciente no início apresenta apenas diminuição da taxa de filtração glomerular, em outros a presença de albumina na urina, determinando a instalação da nefropatia diabética. O surgimento da ND implica em aumento no risco cardiovascular".

No Brasil, a ND é a segunda doença responsável pela terapia de substituição renal ${ }^{5}$. 0 impacto da diálise na qualidade de vida do paciente causa limitações e prejuízos na saúde mental, física e funcional. A necessidade de manter uma dieta específica, a permanência do cateter para facilitar o acesso vascular ou da fístula arteriovenosa afeta a autoestima do paciente, gerando sentimentos de insegurança e insatisfação, comprometendo a eficiência do tratamento³.

O paciente diabético com necessidade de terapia de substituição renal tem três opções: hemodiálise, diálise peritoneal e transplante renal. A diálise peritoneal melhorou a sobrevida nos pacientes quando comparada à hemodiálise, no entanto, a mortalidade nesses pacientes permanece elevada. Ao longo dos anos, a sobrevida dos pacientes com DM2 em diálise é baixa, além disso, conforme a doença evolui aumenta o risco de morte por cardiopatia isquêmica 5 .

Os fatores de risco relacionados ao desenvolvimento e a progressão dessa complicação podem ser genéticos ou não, sendo os mais descritos a hipertensão arterial sistêmica e a hiperglicemia. Outros fatores envolvidos que aumentam o risco são os hábitos de vida, o tempo da doença, dislipidemia, tabagismo, obesidade, predisposição genética e a retinopatia diabética ${ }^{6,7}$.

Estudos recentes têm demonstrado evidências de nefroproteção em todo espectro da função renal em pacientes diabéticos tratados com fármacos da classe dos inibidores da SGLT2, como a dapagliflozina, sugerindo que devem ser oferecidos de rotina para os pacientes com diabetes tipo 2 em risco de doença renal progressiva ${ }^{8}$. Os resultados desses estudos são de grande importância e podem alterar o paradigma da doença renal do diabetes. 
Infere-se então a importância do diagnóstico precoce da complicação renal, o controle da DM e a escolha do melhor tratamento para prevenção ou impedir a progressão da doença. Portanto neste trabalho foram abordados como é realizado o diagnóstico da doença renal do diabetes e as formas de prevenção, com ênfase nos medicamentos mais recentes e promissores. Apesar das recentes e crescentes descobertas de fármacos com efeito nefroprotetor, o tratamento não se limita à medicação. A recomendação da mudança no hábito de vida, da mudança na alimentação, controle da glicemia, HAS e dislipidemia, a prática de exercício físico e abandono do tabagismo são importantes no tratamento do paciente.

\section{MÉTODO}

Trata-se de um estudo exploratório no qual foi realizada uma revisão sistemática da literatura. Para o presente trabalho foi feita uma revisão do conhecimento disponível em bases de dados como Scientific Electronic Library Online (Scielo), Pubmed, Latino-Americana de informação bibliográfica em ciências da saúde (LILACS), Medical Literature Analysis and Retrieval System Online (MEDLINE) e Biblioteca Virtual em Saúde (BVS).

Nas bases de dados selecionadas foram pesquisados em periódicos indexados os termos delimitadores de nefropatias diabéticas, complicações do diabetes e inibidores do transportador 2 de sódio-glicose.

Os acessos às bases de dados científicos ocorreram durante os meses de abril de 2020 e março de 2021. Inicialmente foram selecionados 36 artigos publicados no período de 2001 até outubro de 2020. Foi necessário utilizar artigos publicados recentemente para conterem resultados de estudos pertinentes ao tema deste trabalho. Após a análise dos artigos foi necessário descartar alguns por motivos de não serem atualizados e não conterem informações significativas para este trabalho.

Definiram-se então como critérios de inclusão somente artigos publicados nos últimos 10 anos (2010 a 2020) em língua portuguesa e inglesa. Os critérios de exclusão foram artigos publicados em outros idiomas, artigos com referências desatualizadas e artigos com informações não relevantes ao tema deste trabalho.

Dessa forma, o presente estudo foi feito com base em 19 artigos compreendidos no período de 2010 a outubro de 2020. Entre os artigos que obedeceram aos critérios de inclusão, 13 deles são na língua portuguesa e 06 deles são na língua inglesa.

Além dos artigos mencionados, foram utilizados a Diretriz Nacional da Sociedade Brasileira de Diabetes do ano de 2019/2020, um posicionamento do ano de 2016 da Sociedade Brasileira de Diabetes em conjunto com a Sociedade Brasileira de Endocrinologia e Metabologia e a Sociedade Brasileira de Nefrologia referente ao tema e uma Diretriz Internacional publicada na língua inglesa em Outubro de 2020, pela Kidney Disease Improving Global Outcomes (KDIGO).

A Figura 1 mostra o total de artigos utilizados para esta revisão sistemática e os critérios de exclusão aplicados. 
Figura 1: Fluxograma dos critérios de exclusão dos artigos utilizados como referências.

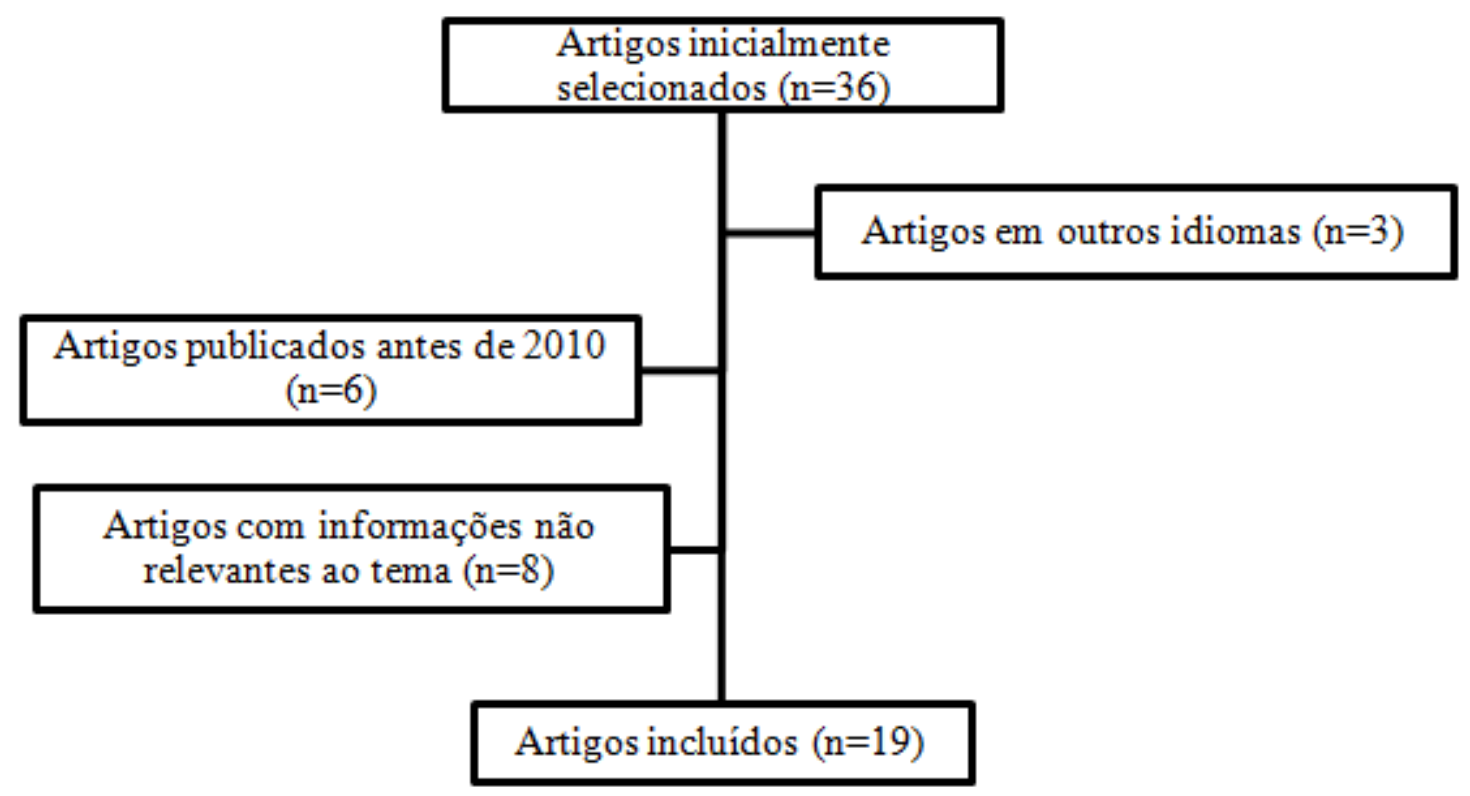

\section{RESULTADOS}

O grande marcador da nefropatia diabética é a albuminúria. Quanto maior o tempo exposto a essa proteinúria maior será a perda da função renal do paciente 4 . 0 diagnóstico precoce é uma ferramenta importante no curso da nefropatia diabética e quando feito nas fases iniciais permite a instituição de tratamentos eficazes que protegem a função renal do paciente ${ }^{9}$.

O rastreamento desta complicação é de grande importância na prevenção de desfechos desfavoráveis ${ }^{10}$. Na prática clínica, para o rastreamento da ND deve ser solicitado um exame de creatinina para estimar a taxa de filtração glomerular e uma coleta de exame de urina simples (EAS). Se o EAS apresentar proteína negativa deve-se proceder investigando a albuminúria em 2 de 3 amostras. 0 resultado negativo da albuminúria implica na vigilância anual deste paciente ${ }^{2}$.

O paciente portador de ND nas fases iniciais é, na maioria das vezes, assintomático ou oligossintomático, o que exige alta suspeição dos médicos em pacientes com fatores de risco ${ }^{10}$. 0 encaminhamento tardio ao especialista está relacionado à maior mortalidade ${ }^{11}$.

O tratamento da doença renal do diabetes não se restringe a medicamentos. O controle glicêmico é fundamental, bem como o controle da pressão arterial e dislipidemia, a prática de exercício físico, alimentação saudável e cessar 0 tabagismo nos pacientes com esse hábito ${ }^{9}$. Em todas as fases do tratamento a modificação do estilo de vida deve ser recomendada ${ }^{12}$.

Os pacientes que apresentam albuminúria elevada devem ser tratados com IECA (inibidores da enzima conversora de angiotensina) ou BRA (bloqueador do receptor da angiotensina), não devendo estes serem usados de 
forma associada ${ }^{13}$. 0 objetivo no uso dessa classe de medicamentos é reduzir a proteinúria ao provocar a vasodilatação da arteríola eferente e diminuir a pressão intraglomerular ${ }^{14}$. Esses medicamentos devem ser utilizados em todos os pacientes com nefropatia diabética e em todos os pacientes hipertensos que são diabéticos².

Aos pacientes que apresentam proteinúria deve ser orientada a restrição protéica com alvo de $0,8 \mathrm{~g} / \mathrm{kg} / \mathrm{dia}$ devendo ser prescrita com cautela e acompanhamento, uma vez que pode causar desnutrição. 0 controle da dislipidemia é importante no tratamento da ND, por mecanismos ainda desconhecidos que ocasionam a redução da proteinúria, além da proteção de eventos cardiovasculares ${ }^{5}$.

Além dessas abordagens terapêuticas citadas há uma classe de medicamentos com resultados promissores na prevenção da nefropatia diabética, os inibidores da SGLT2, alvo de diversos estudos, alguns ainda em andamento, outros com resultados já comprovados.

Os inibidores da SGLT2 têm mostrado resultados satisfatórios em diversos ensaios clínicos, demonstrando evidências na redução da mortalidade cardiovascular, redução da albuminúria, manutenção em longo prazo da taxa de filtração glomerular e diminuição das hospitalizações por insuficiência cardíaca' ${ }^{15}$.

Três grandes ensaios clínicos estudaram os benefícios cardiovasculares e renais dos inibidores de SGLT2 e demonstraram que de forma geral os inibidores da SGLT2 são medicamentos seguros e bem tolerados. 0 principal efeito adverso relatado foram as infecções genitais fungícas como a vulvovaginite e a balanopostite ${ }^{16}$.

Um dos ensaios é o EMPA-REG que estudou os efeitos da empagliflozina em um ensaio clínico duplo-cego no qual 7028 pacientes com DM2 e doença cardiovascular estabelecida foram divididos em 3 grupos que receberam placebo, $10 \mathrm{mg} \mathrm{e} 25 \mathrm{mg}^{16}$. Os pacientes participantes, de 42 países, foram acompanhados por 3 anos. A empaglifozina foi adicionada à terapia individualizada de cada paciente, ou seja, não substituiu os medicamentos que o paciente já fazia uso. Os resultados mostraram que nos pacientes que fizeram o uso da droga, independente da dose, houve uma redução significativa da mortalidade cardiovascular, com redução de 32\% da mortalidade geral e diminuição de 35\% das internações por insuficiência cardíaca ${ }^{16,17}$. Nos casos de infarto agudo do miocárdio e acidente vascular cerebral não houve digerenças significativas nos desfechos com o uso do fármaco. 0 estudo prosseguiu avaliando-se os resultados renais nesses pacientes os quais a maioria, cerca de $75 \%$, apresentavam uma eTFG (taxa de filtração glomerular estimada) acima de $60 \mathrm{ml} / \mathrm{min} / 1,73 \mathrm{~m}^{2}$ e cerca de $40 \%$ apresentavam relação albuminúria/creatinina acima de $30 \mathrm{mg} / \mathrm{g}$. Os resultados evidenciaram que os pacientes tratados com empaglifozina demonstraram redução da progressão da doença renal crônica com diminuição da progressão da eTFG de até $4 \mathrm{ml} / \mathrm{min} / 1,73 \mathrm{~m}^{2}$ nas primeiras quatro semanas de tratamento enquanto no grupo que fez uso de placebo houve redução progressiva da eTFG durante o período do estudo $0^{16,17}$.

O segundo estudo é o CANVAS, um ensaio clínico multicêntrico randomizado que envolveu 10.142 pacientes para avaliar a eficácia da canagliflozina primeiramente nos desfechos da mortalidade cardiovascular, IAM não fatal e AVC 
não fatal ${ }^{16}$. Partiparam deste estudo pacientes com DM2 e doença cardiovascular estabelecida ou que apresentavam fatores de risco para doenças cardiovasculares ${ }^{18}$. Este estudo randomizado foi dividido em dois desenhos : Canvas e Canvas-R. O primeiro separou os pacientes em três grupos: placebo, canagliflozina $100 \mathrm{mg}$ e canaglifozina $300 \mathrm{mg}$, ao passo que o segundo dividiu os pacientes em dois grupos: placebo e canagliflozina 100mg (com a possibilidade dessa dose ser aumentada para 300mg durante o acompanhemento). 0 canvas demonstrou diminuição da hospitalização por insuficiência cardíaca e da mortalidade cardiovascular ${ }^{18}$. No que se refere à função renal, os pacientes que fizeram o uso do fármaco apresentaram redução da progressão da albuminúria, diminuição da necessidade de terapia de substituição renal, redução da progressão da eTFG em 40\% e consequentemente redução de óbitos por causas renais nesses pacientes ${ }^{16}$. Em relação aos efeitos adversos apresentados pela canagliflozina foram descritos maior incidência de infecções genitais, maior diurese osmótica e redução do volume de sangue nos grupos que fizeram uso da droga. Além disso, nos pacientes que fizeram uso da canagliflozina foram observados uma maior incidência de amputações e fraturas, o que não teve o mecanismo totalmente compreendido, mas que exige mais estudos e cautela em pacientes suscetíveis, devendo estes serem monitorados quanto a esses riscos ${ }^{16}$.

ODECLARE-TIMI 58 é um outro ensaio clínico multicêntrico randomizado que avaliou as ações da dapagliflozina. Participaram deste estudo 17.160 pacientes com DM2 e doença cardiovascular já estabelecida ou pacientes que apresentavam alto risco cardiovascular. Esses pacientes foram didividos em dois grupos (placebo e dapagliflozina $10 \mathrm{mg}$ ) e acompanhados por cerca de 4 anos $^{16}$. Os resultados mostraram que os pacientes que receberam a droga apresentaram redução na hospitalização por insuficiência cardíaca e na mortalidade cardiovascular, porém não mostraram redução significativa na incidência de efeitos cardiovasculares maiores ${ }^{16,18}$. Na análise dos desfechos renais os pacientes que utilizaram dapagliflozina apresentaram redução na incidência de doença renal em estágio terminal, diminuição maior que $40 \%$ na progressão da eTFG e redução das mortes por insuficiência renal ${ }^{18}$. Os efeitos adversos encontrados nesse grupo de pacientes foram a infecção genital e a cetoacidose diabética ${ }^{16}$.

A limitação dos estudos apresentados se deve ao fato de que os pacientes selecionados apresentavam pouca ou nenhuma albuminúria, eTFG normal ou timidamente reduzida, ou seja, indivíduos com baixo risco de progressão renal, não demonstrando se tem eficácia em pacientes que já apresentam lesões renais ${ }^{16}$.

O estudo CREDENCE, em oposição aos estudos já mencionados, randomizou 4.401 pacientes que atendessem aos seguintes critérios: pacientes com DM2 com eTFG entre 30 a $90 \mathrm{ml} / \mathrm{min} / 1,73 \mathrm{~m}^{2}$, UACR entre 300 a $5000 \mathrm{mg} / \mathrm{g} \mathrm{e}$ pacientes que tivessem dose estável e ideal de inibidores da enzima de conversão da angiotensina ou receptor da angiotensina bloqueador ${ }^{16}$. Os pacientes selecionados foram divididos em dois grupos (placebo e $100 \mathrm{mg}$ de canagliflozina) e acompanhados por 2,6 anos. Os resultados mostraram positivos desfechos renais com diminuição de $30 \%$ da doença renal em estágio terminal, da duplicação da creatinina sérica, mortalidade cardiovascular e renal. Neste estudo não houve aumento na incidência de amputações e fraturas ${ }^{16}$. 
O DAPA-CKD, ensaio clínico que analisou o efeito da dapagliflozina em pacientes com doença renal crônica, com ou sem diabetes tipo 2 utilizou 4.304 participantes com uma eTFG de 25 a $75 \mathrm{ml} / \mathrm{min} / 11,73$ m² e uma relação albumina / creatinina urinária de $200 \mathrm{mg}$ a $5000 \mathrm{mg}$. Esses pacientes foram divididos entre os que receberam placebo e dapagliflozina $10 \mathrm{mg}$ uma vez ao dia ${ }^{8}$. Os resultados primários apontaram um declínio sustentado na progressão da TFG estimada de pelo menos 50\%, doença renal em estágio final ou morte por causas renais ou cardiovasculares. Tanto pacientes com DM2 quanto os sem a patologia apresentaram efeitos semelhantes com o uso do fármaco. 0 estudo concluiu que o conhecido perfil de segurança da dapagliflozina foi confirmado e que os pacientes que fizeram uso da dapagliflozina tiveram benefícios renais como o declínio sustentado na progressão da TFG estimada de pelo menos $50 \%$, a diminuição da doença renal em estágio terminal e a morte por problemas renais ou cardiovasculares ${ }^{8}$.

Os resultados dos ensaios clínicos apresentados afirmam a segurança dos inibidores do SGLT2 no tratamento de diabetes mellitus, com evidências de nefroproteção ${ }^{8}$. Os inibidores da SGLT2 reduziram o risco de necessidade de diálise, transplante, ou morte devido a doença renal em indivíduos com diabetes tipo 2 e, além disso, conferiu proteção ao paciente contra lesões renais agudas ${ }^{8}$.

São medicamentos bem tolerados, porém deve-se atentar aos possíveis efeitos adversos citados como as amputações, fraturas, cetoacidose diabética, depleção de volume sanguíneo e as infecções genitourinárias ${ }^{8,18}$.

Em relação aos valores monetários das drogas apresentadas nos estudos, a empaglifozina, dapaglifozina e canaglifozina são medicamentos de custo elevado, principalmente se comparadas à metformina. A tabela abaixo apresenta a faixa de preço dos medicamentos, pesquisados no site de duas redes de farmácia, sendo elas Drogasil e Ultrafarma ${ }^{19,20}$.

Tabela 1: Alto custo dos inibidores da SGLT-2 quando comparados ao valor da metformina.

Faixa de preço dos medicamentos citados nos estudos

\begin{tabular}{|c|c|c|c|c|}
\hline \multicolumn{2}{|c|}{ Medicamento } & Nome comercial & $\begin{array}{l}\text { Caixa com } 30 \\
\text { comprimidos }\end{array}$ & Faixa de preço \\
\hline \multirow{2}{*}{\multicolumn{2}{|c|}{ Empaglifozina }} & Jardiance & $10 \mathrm{mg}$ & $\mathrm{R} \$ 220,08$ - R\$224,99 \\
\hline & & & $25 \mathrm{mg}$ & $\mathrm{R} \$ 220,08$ - R\$224,99 \\
\hline Dapaglifozina & & Forxiga & $10 \mathrm{mg}$ & $\mathrm{R} \$ 122,99-\mathrm{R} \$ 176,75$ \\
\hline \multirow[t]{2}{*}{ Canaglifozina } & & Invokana & $100 \mathrm{mg}$ & $\mathrm{R} \$ 126,49-\mathrm{R} \$ 177,32$ \\
\hline & & & $300 \mathrm{mg}$ & $\mathrm{R} \$ 160,99$ - R\$227,65 \\
\hline Cloridrato & de & Metformina & $500 \mathrm{mg}$ & $\mathrm{R} \$ 4,73-\mathrm{R} \$ 9,43$ \\
\hline Metformina & & & $850 \mathrm{mg}$ & $\mathrm{R} \$ 4,99-\mathrm{R} \$ 5,97$ \\
\hline Dapaglifozina & + & XigDuo XR & $5 / 1000 \mathrm{mg}^{*}$ & $\mathrm{RS} 148,35$ - R\$179,52 \\
\hline Cloridrato & de & & $10 / 1000 \mathrm{mg}$ & $\mathrm{R} \$ 143,99$ - R\$179,59 \\
\hline
\end{tabular}

Metformina

${ }^{*}$ Caixa com 60 comprimidos 
São aguardados mais estudos a respeito da segurança e dos benefícios dos inibidores da SGLT2, principalmente em pacientes com a função renal comprometida. Os resultados desses estudos podem melhorar as perspectivas futuras do tratamento da doença renal do diabetes. O que se pode afirmar por enquanto é que os benefícios são maiores que os riscos, devendo prevalecer a segurança do paciente, a individualidade de cada caso e a boa conduta médica com o objetivo de identificar complicações e situações adversas de maneira precoce. Essa classe de medicamentos já é utilizada na prática clínica no tratamento do DM2, tem como limitação o alto custo no Brasil, sua prescrição deve ser incentivada e a relação custo-benefício apresentada ao paciente.

\section{DISCUSSÃO}

A incidência da doença renal do diabetes vem aumentando no Brasil e é atualmente um problema de saúde pública mundial. O prognóstico da doença é ruim, os custos do tratamento são altos e a patologia afeta diretamente a qualidade de vida do paciente e de sua família ${ }^{21}$. As consequências implicadas podem ser prevenidas ou retardadas se a doença for reconhecida precocemente, portanto o momento do diagnóstico é a chave do tratamento e prevenção da doença renal do diabetes ${ }^{10}$.

Os termos nefropatia diabética e doença renal do diabetes, apesar de muitas vezes serem usados como sinônimos apresentam diferenças. A nefropatia diabética é instituída quando o paciente apresenta uma disfunção crônica microvascular que resulta em perda progressiva da função renal devido a alterações estruturais, provocadas pela doença subjacente, que provocam a albuminúria ${ }^{6}$. Alguns pacientes no início da doença não apresentam proteinúria e tem como alteração apenas a diminuição da taxa de filtração glomerular, nesses casos, o termo correto a ser utilizado é a doença renal do diabetes. Uma vez que a doença é subdiagnosticada, a maioria dos pacientes já são diagnosticados com nefropatia diabética ${ }^{22}$.

O ideal é que pacientes diabéticos tenham a função renal avaliada periodicamente, com o objetivo de detectar precocemente alterações de forma a evitar a instalação da nefropatia ou postergar a evolução desta complicação. Pacientes com DM2 devem ser rastreados para ND logo após o diagnóstico, uma vez que os mesmos podem apresentar a doença há tempo sem terem o conhecimento. Já no caso de pacientes com DM1 o rastreamento deve ser feito após 5 anos do diagnóstico, exceto nos casos de pacientes com DM descompensada ou que estão no período da puberdade

O diagnóstico da ND é feito, segundo a American Diabetes Association (ADA), principalmente pela diminuição da taxa de filtração glomerular (TFG) e pela elevada excreção urinária de albumina (EUA). A estimativa da taxa de filtração glomerular é feita avaliando-se anualmente a creatinina sérica pela equação de Chronic Kidney Disease Epidemiology Collaboration (CKD-EPI), que é realizada através de um cálculo que correlaciona a dosagem de creatinina com a idade, 
o gênero e a etnia do paciente ${ }^{6}$. A forma mais fidedigna de se calcular a TGF é através da medida da depuração renal de marcadores exógenos ou por componentes radioativos, no entanto, o alto custo, a complexidade e a radioatividade inviabilizam seu uso rotineiro na prática clínica, restringindo seu uso a situações especiais. No manejo dos pacientes diabéticos a fim de estimar a TFG, a opção rotineira é medir a concentração sérica de creatinina ${ }^{2}$. Diversos marcadores podem ser utilizados no rastreio precoce da ND, no entanto a presente revisão tem enfoque nos principais métodos utilizados na realidade do acompanhamento destes pacientes.

Segundo as Diretrizes da Sociedade Brasileira de Diabetes de 2019, a classificação dos níveis da Taxa de Filtração Glomerular é feita conforme a tabela abaixo:

Tabela 2: EUA elevada tem correlação com danos renais.

\begin{tabular}{|c|c|c|}
\hline Estágios & Descrição & TFG $\left(\mathrm{mL} / \mathrm{min} / 1,73 \mathrm{~m}^{2}\right)$ \\
\hline 1 & TFG normal ou elevada & $\geq 90$ \\
\hline 2 & TFG levemente reduzida & 60 a 89 \\
\hline $3^{a}$ & Moderada redução da TFG & 45 a 59 \\
\hline $3 \mathrm{~B}$ & Redução marcada da TFG & 30 a 44 \\
\hline 4 & Redução grave da TFG & 15 a 29 \\
\hline 5 & Insuficiência Renal & $<15$ \\
\hline
\end{tabular}

Esses valores não devem ser avaliados isoladamente. Pacientes com índice sugestivo de ND devem ser avaliados outros fatores como presença de proteinúria na urina, sedimentos urinários, como cilindros hemáticos, leucocitários e acantócitos, e manifestações clínicas que podem sugerir outra doença sistêmica².

A excreção urinária de albumina é realizada através da amostra de urina isolada com um método padronizado de 24 horas, sendo considerado um exame rápido e barato. A amostra deve ser confirmada em duas medidas em um intervalo de até seis meses, devido à variação diária da EUA. A EUA tem como ponto de corte diagnóstico da doença renal do diabetes o valor de $20 \mu \mathrm{g} / \mathrm{min}$ ou $30 \mathrm{mg} / 24 \mathrm{~h}$, sendo considerados normais valores abaixo destes. Uma outra forma é a quantificação em três classes: normal (A1), elevada (A2) e muito elevada (A3)

Alguns fatores podem elevar a EUA e, portanto, devem ser considerados, sendo eles: intensa atividade física, infecção do trato urinário, glicemia não controlada, hipertensão arterial sistêmica não controlada, obesidade mórbida, insuficiência cardíaca descompensada, doença aguda ou febre, hematúria, sobrecarga protéica ou hídrica, menstruação, 
leucorréia e gestação ${ }^{6}$.

Segundo a Diretriz de 2018 da Sociedade Brasileira de Diabetes a classificação dos níveis de excreção urinária de albumina é feita da seguinte forma ${ }^{6}$ :

Tabela 3: Maior índice de EUA está associado com maior lesão renal.

\section{Classificação dos níveis da Excreção Urinária de Albumina}

$\begin{array}{lllll}\text { Estágios } & \begin{array}{l}\text { Urina de } 24 h \\ (\mathbf{m g} / \mathbf{2 4 h})\end{array} & \begin{array}{l}\text { Albumina/Creatinina } \\ (\mathbf{m g} / \mathbf{g})\end{array} & \begin{array}{l}\text { Concentração } \\ (\mathbf{m g} / \mathbf{L})\end{array} \\ \text { A1 } & <20 & <30 & <30 & <17 \\ \text { A2 } & 20 \text { a } 199 & 30 \text { a } 299 & 30 \text { a } 299 & 17 \text { a } 173 \\ \text { A3 } & \geq 200 & \geq 300 & \geq 300 & \geq 174\end{array}$

A avaliação da EUA tem algumas limitações, estudos recentes demonstraram que mesmo níveis considerados normais podem conferir risco de progressão de doença renal e cardiovascular. Além disso, ocorre uma subestimação dos valores de albumina, uma vez que uma significativa quantidade de albuminúria não é detectada pelos métodos convencionais $^{10}$. Uma alternativa a essa limitação seria a cromatografia líquida de alta eficiência (HPLC), um método que mede a fração não-imunorreativa de albuminúria, tornando o resultado mais fidedigno, mas que tem pouco uso na prática clínica. Ainda referente às limitações deste exame sabe-se que pacientes com valores dentro da normalidade e, portanto considerados normoalbuminúricos podem já apresentar diminuição da TFG. Por essa razão, no rastreamento de ND não se deve avaliar o valor de um único exame isoladamente. Recomenda-se que a TFG seja estimulada, ainda que o paciente apresente valores normais EUA ${ }^{6}$.

A importância do rastreamento precoce é explicada pela compreensão da história natural da nefropatia diabética. O diagnóstico nas fases iniciais da doença permite a instituição do tratamento com resultados benéficos ao paciente, preservando sua função renal e postergando a evolução da patologia ${ }^{14}$.

Em relação à terapêutica da doença renal do diabetes, o controle glicêmico apresenta muita eficiência no controle e na sua prevenção. No tratamento inicial do paciente diabético, a primeira opção para controle da glicemia, segundo a ADA é o uso do hipoglicêmico oral, metformina associado a mudanças no estilo de vida como dieta e a prática de exercício físico. Essa escolha é justificada pelo baixo custo do medicamento, disponível no SUS, e pelos seus benefícios cardiovasculares e metabólicos já comprovados².

Em pacientes com doença cardiovascular estabelecida a ADA recomenda como tratamento auxiliar o uso dos hipoglicemiantes orais da classe dos inibidores da SGLT2 (representados pela dapagliflozina, empagliflozina e canagliflozina) ou o medicamento da classe das incretinas, o hormônio agonista do peptídeo 1 semelhante ao glucagon- 
GLP-1 (representados principalmentepela liraglutida, exenatida e semaglutida) ${ }^{23}$.

Em pacientes hipertensos e diabéticos os IECA têm demonstrado benefício na prevenção da doença renal e cardioproteção, sendo a medicação de primeira escolha como anti-hipertensivo nesses pacientes².

Apesar da discussão acerca do efeito nefroprotetor dos inibidores da SGLT2 parecerem recentes, a inibição renal da proteína teve origem na década de 1980 e desde então foi evoluida e experimentada até chegar nos medicamentos disponíveis hoje ${ }^{16}$

A SGLT2 é uma proteína presente na membrana apical das células do túbulo contorcido proximal do néfron e tem a função de cotransporte de sódio e de glicose. A enzima é responsável por reabsorver cerca de $90 \%$ da glicose filtrada na região. A classe de medicamentos inibidores da SLGT2 tem como ação o bloqueio desta enzima, causando a diminuição da reabsorção tubular de glicose e consequentemente aumentando a glicosúria. Dessa forma, a droga diminui a glicemia em jejum e pós prandial, uma vez que age de modo independente da ação da insulina. Como consequência da glicosúria provocada, um dos efeitos colaterais desagradáveis dos inibidores da SGLT2 é o aumento das infecções genitais e do trato urinário ${ }^{24}$.

Além da ação no controle glicêmico, os inibidores de SGLT2 tem benefícios extraglicêmicos como a redução de peso, controle da pressão arterial, diminuição dos triglicerídeos e do ácido úrico e, o ponto mais importante neste estudo, o potencial de retardar a doença renal em pacientes com a doença renal do diabetes ${ }^{24}$.

Os inibidores da SGLT2 são os medicamentos com o maior avanço farmacológico em nefrologia desde os bloqueadores do sistema renina angiotensina aldosterona. Estudos recentes sobre essa classe de medicamentos podem revolucionar o tratamento e a perspectiva na sobrevida de pacientes com DM2. Considerando a alta morbimortalidade associada a DM e as complicações acerca dessa patologia torna-se cada vez mais necessário terapêuticas mais eficazes que permitam a sobrevida e a qualidade de vida desses pacientes.

\section{CONSIDERAÇÕES FINAIS}

A albuminúria é o primeiro marcador laboratorial de nefropatia diabética, devendo ser investigada juntamente com a alteração na taxa de filtração glomerular. Tendo em vista que o portador de doença renal do diabetes na maioria das vezes é assintomático, é fundamental o acompanhamento periódico dos pacientes diabéticos após certo tempo de diagnóstico da doença. O maior benefício da reversão da ND é nas fases I e II, consideradas iniciais, mas o paciente deve ser tratado em todas as fases. O tratamento desta complicação é complexo e envolve mudanças nos hábitos de vida e uso de medicamentos. Os estudos mais recentes acerca dos inibidores da SGLT2, usado para controle glicêmico nos pacientes diabéticos, evidenciaram sua segurança e eficiência na proteção renal e cardiovascular, devendo estes 
(dapaglifozina $10 \mathrm{mg}$, canaglifozina $100 \mathrm{mg}$ e $300 \mathrm{mg}$ e empaglifozina $10 \mathrm{mg}$ e $25 \mathrm{mg}$ ) serem prescritos a esses pacientes. Os benefícios dessa classe de medicamentos superam os riscos, mas uma limitação ao seu uso rotineiro na prática clínica é o alto custo. A prevenção e o diagnóstico precoce em conjunto com o tratamento adequado da doença promovem benefícios ao paciente, diminui os desfechos desfavoráveis e reduz os custos relacionados à saúde, tanto para o paciente como para o sistema público de saúde.

\section{REFERÊNCIAS}

1. Amorim RG, Guedes GS, Vasconcelos SM, Santos JC. Doença Renal do Diabetes: Cross-Linking entre Hiperglicemia, Desequilíbrio Redox e Inflamação. Arq. Bras. Cardiol. [online]. 2019, vol.112, n.5, pp.577-587.

2. Sociedade Brasileira de Diabetes. Diretrizes da Sociedade Brasileira de Diabetes 2019-2020. 2019.

3. Pilger C, Rampari, EM, Waidman, MA, Carreira L. Hemodiálise: seu significado e impacto para a vida do idoso. Esc. Anna Nery [online]. 2010, vol.14, n.4, pp.677-683.

4. Alves CM, Lima CS, Oliveira FJ. Nefropatia diabética: avaliação dos fatores de risco para seu desenvolvimento. Rev Bras Clin Med., São Paulo, p. 97-100, mar. 2011.

5. Sociedade Brasileira de Diabetes. Sociedade Brasileira de Nefrologia. Posicionamento Oficial Tripartite $n$. 01/2016. Prevenção, Diagnóstico e Conduta terapêutica na doença renal do diabetes [Internet] (Acesso em 2020, outubro).

6. Maciel RO, Vasconcelos MR, Andrade CR. Nefropatia diabética - incidência e fatores de risco associados. Brazilian Journal Of Health Review, [s.I.], v. 2, n. 4, p. 3808-3823, 2019.

7. Flor LS, Campos MR. Prevalência de diabetes mellitus e fatores associados na população adulta brasileira: evidências de um inquérito de base populacional. Revista Brasileira de Epidemiologia, v. 20, p. 16-29, 2017.

8. Heerspink HJ, Stefánsson BV, Rotter RC, Chertow GM, Greene T, Hou, FF, et al. Dapagliflozin in Patients with Chronic Kidney Disease. New England Journal Of Medicine, [S.L.], v. 383, n. 15, p. 1436-1446, 8 out. 2020.

9. Viana MR, Rodriguez TT. Complicações cardiovasculares e renais no diabetes mellitus. Revista de ciências médicas e biológicas, v. 10, n. 3, p. 290-296, 2011.

10. Bastos MG, Kirsztajn GM. Doença renal crônica: importância do diagnóstico precoce, encaminhamento imediato e abordagem interdisciplinar estruturada para melhora do desfecho em pacientes ainda não submetidos à diálise. Brazilian Journal of Nephrology, v. 33, n. 1, p. 93-108, 2011.

11. Dallacosta FM, Dallacosta H, Mitrus L. Detecção precoce de doença renal crônica em população de risco. Cogitare Enfermagem, v. 22, n. 1, 2017. 
12. Bertoluci MC, Salles JE, Nunes JS, Pedrosa HC, Moreira RO, Duarte RM et al. Portuguese-Brazilian evidence-based guideline on the management of hyperglycemia in type 2 diabetes mellitus. Diabetology \& Metabolic Syndrome, v. 12, p. 1-30, 2020.

13. Júnior JM, Suassuna JH. O acometimento renal na hipertensão arterial e diabetes mellitus tipo 2: como identificar e prevenir-A visão do nefrologista. Revista Hospital Universitário Pedro Ernesto, v. 12, 2013.

14. Pereira JL, Ferreira NA, Gabriel D, Silva JE. Microalbuminúria: aviso de alerta às nefropatias diabéticas. 2010.

15. Foresto RD, Pestana JO. Novel treatment options for chronic kidney disease complications. Rev. Assoc. Med. Bras., São Paulo, v. 66, 13 jan. 2020.

16. Gonzalez DE, Foresto RD, Ribeiro AB. SGLT-2 inhibitors in diabetes: a focus on renoprotection. Revista da Associação Médica Brasileira, [s.l.], v. 66, n. 1, p. 17-24, 2020. FapUNIFESP (SciELO).

17. Sridhar VS, Dubrofsky L, Boulet J, Cherney DZ. Making a case for the combined use of SGLT2 inhibitors and GLP1 receptor agonists for cardiorenal protection. Brazilian Journal of Nephrology, n. AHEAD, 2020.

18. Martins EB, Lima EG, Pitta FG, Carvalho LN, Queiroz TD, Júnior CV. Pharmacological therapy and cardiovascular risk reduction for type 2 diabetes. Revista da Associação Médica Brasileira, v. 66, n. 9, p. 1283-1288, 2020.

19. Drogasil, BRASIL, 2021. Disponível em: https://www.drogasil.com.br/ Acesso em 17 fev. 2021.

20. Ultrafarma, BRASIL, 2021. Disponível em: https://www.ultrafarma.com.br/ Acesso em 17 fev. 2021.

21. Bastos MG, Bregman R, Kirsztajn GM. Doença renal crônica: frequente e grave, mas também prevenível e tratável. Revista da Associação Médica Brasileira, v. 56, n. 2, p. 248-253, 2010.

22. Madeira MP, Carvalho MM, Gadelha DD, Queiroz PC, Fernandes VO, Montenegro RM. Diabetes e Doença renal. Ver Med UFC. 2019 jan-mar;59(1):75-76.

23. Kidney Disease: Improving Global Outcomes (KDIGO) Diabetes Work. KDIGO 2020 Clinical Practice Guideline for Diabetes Management in Chronic Kidney Disease. Kidney International, v. 98, n. 4S, p. S1-S115, 2020.

24. Ferreira VA, Campos SM. Avanços farmacológicos no tratamento do diabetes tipo 2. Brazilian Journal of Surgery and Clinical Research, v. 8, n. 3, p. 72-78, 2014. 